\title{
Monte Carlo calculated CT numbers for improved heavy ion treatment planning
}

\author{
Sima Qamhiyeh, \\ Anna Wysocka-Rabin, \\ Oliver Jäkel
}

\begin{abstract}
Better knowledge of CT number values and their uncertainties can be applied to improve heavy ion treatment planning. We developed a novel method to calculate CT numbers for a computed tomography (CT) scanner using the Monte Carlo (MC) code, BEAMnrc/EGSnrc. To generate the initial beam shape and spectra we conducted full simulations of an X-ray tube, filters and beam shapers for a Siemens Emotion CT. The simulation output files were analyzed to calculate projections of a phantom with inserts. A simple reconstruction algorithm (FBP using a Ram-Lak filter) was applied to calculate the pixel values, which represent an attenuation coefficient, normalized in such a way to give zero for water (Hounsfield unit (HU)). Measured and Monte Carlo calculated CT numbers were compared. The average deviation between measured and simulated CT numbers was $4 \pm 4 \mathrm{HU}$ and the standard deviation $\sigma$ was $49 \pm 4$ HU. The simulation also correctly predicted the behaviour of H-materials compared to a Gammex tissue substitutes. We believe the developed approach represents a useful new tool for evaluating the effect of CT scanner and phantom parameters on $\mathrm{CT}$ number values.
\end{abstract}

Key words: X-ray tomography $\bullet$ Monte Carlo (MC) method $\bullet$ treatment planning $\bullet$ hadrontherapy

S. Qamhiyeh

West German Proton Therapy Centre Essen (WPE), Hufelandstraße 55, 45147 Essen, Germany

A. Wysocka-Rabin ${ }^{凶}$

Division of Accelerator Physics,

National Centre for Nuclear Research (NCBJ),

7 Andrzeja Soltana Str., 05-400 Otwock/Świerk, Poland, Tel.: +48 22718 0423, Fax: +48 22779 3481,

E-mail: anna.wysocka@ncbj.gov.pl

\section{O. Jäkel}

Heidelberg Ion-Beam Therapy Centre HIT,

Im Neuenheimer Feld 450, 69120 Heidelberg, Germany

Received: 26 November 2013

Accepted: 8 January 2014

\section{Introduction}

A computer tomography (CT) image is the measurement of X-ray attenuation coefficients in the scanned object. The image can be represented in grey scale. The grey values or $\mathrm{CT}$ numbers represent the attenuation coefficients of the object relative to the attenuation coefficients of water.

$$
\text { CTnumber }=1000 \times\left(\frac{\mu-\mu_{\text {water }}}{\mu_{\text {water }}}\right) \mathrm{HU}
$$

Here, $\mu$ is the linear attenuation coefficient for the scanned object and $\mu_{\text {water }}$ is the linear attenuation coefficient for water, HU is the Hounsfield unit. Empirically, the value of CT numbers is affected by such measurement parameters as the size and material of the phantom used for the CT measurements, the kilovolt potential setting of the X-ray tube $[1,2]$ reconstruction field of view (FOV), elemental composition of the scanned media [1] and its temperature [3].

Previous studies of CT scanners, including empirical models and Monte Carlo (MC) simulations, were conducted in order to minimize radiation dose to the patients or to improve image quality (Bhat et al. [4], Caon et al. [5] and later by Ay et al. [6, 7]).

Calculating the effect of beam shaping filters is an important aspect of these simulations and several methods have been used to simulate CT filters. Atherton and Huda [8] created a look-up table containing information 
on the angle of incidence and the amount of material in the beam path, an approach also taken by Jarry et al. [9]. In these studies, the information was transferred into weighing factors [9] and a look up table of linear attenuation coefficients as a function of angle [8]. Full Monte Carlo simulation of the filter geometry was carried out by Salvado et al. [10] and Tzedakis et al. [11].

In our work, simulations were performed to establish an approach to calculate the accuracy of CT numbers. Our approach included performing Monte Carlo simulations of a CT scanner and phantom with tissue substitutes, and then reconstructing the simulated projections into images with $\mathrm{CT}$ number representation. We validated this approach to calculate CT numbers through a comparison with experimental measurements. The aim was to predict how different parameters affect the uncertainty of the CT numbers and how they indirectly affect the empirical relation between measured CT numbers and the carbon ion range used in treatment planning. We presented initial results of the MC simulation of the $\mathrm{X}$-ray CT scanner and phantom with tissue substitutes in [12]. The effect of the photon spectral distribution and energy fluence across the FOV on CT images was also discussed in the prior article. This paper presents our complete findings.

\section{Materials and methods}

\section{X-ray CT scanner}

A Siemens Emotion CT unit was simulated based on design data provided by Siemens Medical Solutions ${ }^{1}$. The scanner is equipped with a DURA 352-MV X-ray tube, two beam shaping filters (a bow-tie filter made of $\mathrm{Al}$ and a Teflon filter). The detector-arc is made out of 672 elements and equipped with an anti-scatter grid. The material of the detector elements is gadolinium oxysulphide $\left(\mathrm{Gd}_{2} \mathrm{O}_{2} \mathrm{~S}\right)$ for which the energy response function has been published [13].

The X-ray tube of the CT scanner has three nominal voltages: 80,110 and $130 \mathrm{kV}$. However, the air calibration of this particular scanner is maintained by changing the voltage settings of the X-ray tube. The voltage can vary by up to $\pm 10 \mathrm{kV}$.

\section{Substitutes and phantoms}

Electron density substitutes, manufactured by Gammex-RMI (Middletown, WI, USA) were chosen as tissue equivalent substitute materials [14]. Another group of tissue substitutes (H-materials) has been experimentally investigated by Jäkel et al. [15]. H-materials are based on polyethylene and simulate tissue in terms of photon absorption, but their elemental composition contains high percentages of silicon and tin. In the scope of this work, the elemental composition of the Gammex tissue substitutes and $\mathrm{H}$-material was modelled for the calculation of CT numbers.

\footnotetext{
${ }^{1}$ The confidential data were obtained from Siemens within the framework of a collaboration contract signed by "Siemens Medical Systems" and the Medical Physics Department including a nondisclosure agreement.
}

A cylindrical phantom (16 cm diameter) of PMMA was simulated. Gammex tissue substitutes and $\mathrm{H}$-materials were simulated as phantom inserts $(2.8 \mathrm{~cm}$ in diameter). In order to present the simulated results in terms of Hounsfield units, air and water were simulated as reference media. The elemental composition of the simulated materials is presented in Table 1 .

CT numbers of Gammex tissue substitutes were measured using a Siemens Emotion CT scanner. Measurements were done at nominal tube voltages 80,110 and $130 \mathrm{kV}$, with the tube coefficient (mAs) equal to $200 \mathrm{mAs}, 3 \mathrm{~mm}$ slice thickness, H40s reconstruction filter and $287 \mathrm{~mm}$ in diameter FOV. As for the $\mathrm{H}$-materials, the reported CT measurements are those reported by Jäkel et al. [15].

\section{Calculating CT numbers from Monte Carlo simulation of CT scanner and phantom inserts}

$\mathrm{X}$-ray projections of the phantom and inserts were simulated using the MC code BEAMnrc/EGSnrc [16-18]. EGSnrc was chosen because it has the most advanced interaction library for physics modelling of electron, positron and photon interactions in low energy range applications (1-150 keV) [16, 19, 20].

The MC simulation was divided into two parts. The first deals with X-ray production and filtration (CT1). The second deals with the transport of photons through phantoms and tissue substitutes (CT2). Each part was terminated by a scoring plane. Phase space files (PHSP) were calculated at each scoring plane [12].

The phase space file contains information on the energy, position and incidence angles of the photons. However, projection data are required to perform the reconstruction and to acquire $\mathrm{CT}$ numbers. Calculating projections from PHSP involves the sub-processes of sorting the photons into detector elements, accounting for detector response, and finally representing the signal in raw data format for projections. Scatter and beam hardening corrections are applied. Images are reconstructed from many projections using a PC-based FBP algorithm with a Ram-Lak filter. Finally, average CT numbers were calculated by defining regions of interest (ROI) in the reconstructed images and presented in Hounsfield units scale. The calculation chain for CT numbers is shown in Fig. 1.

\section{Simulation of CT unit and phantom with substitutes}

EGSnrc parameters were optimized for low energy interactions. The photon cut-off-energy (PCUT) and the electron cut-off-energy (ECUT) were 0.001 and $0.516 \mathrm{MeV}$, respectively. We used an ECUT of $0.521 \mathrm{MeV}$ to save calculation time in the filters and collimators. Bound Compton scattering, Rayleigh scattering and atomic relaxation effects were turned on during the simulation. The cross-section of all interactions of photons with the simulated materials was created using the code PEGS4 for $A E=0.516 \mathrm{MeV}$ and $A P=0.001 \mathrm{MeV}$. The parameters $A P$ and $A E$ are the low-energy thresholds for the production of secondary Bremsstrahlung photons and knock-on electrons, respectively. 


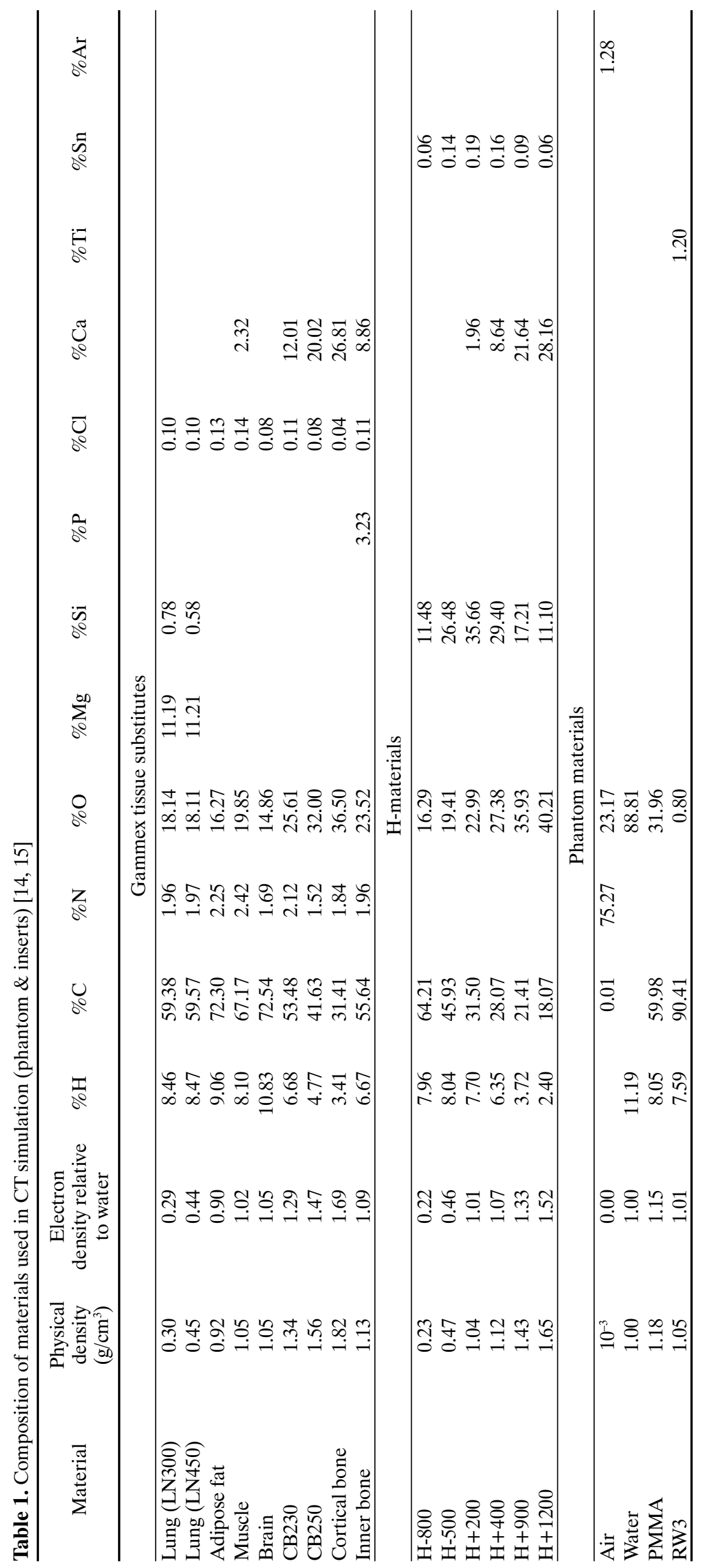




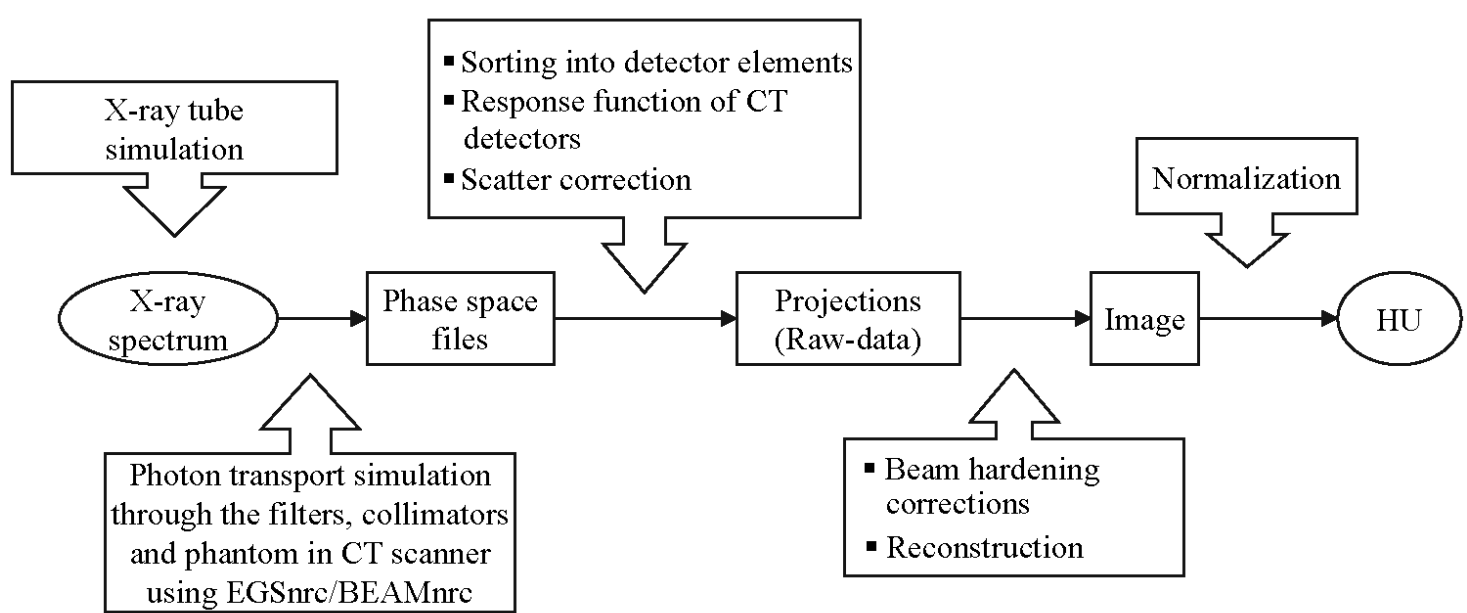

Fig. 1. Workflow to simulate CT numbers taking into consideration the geometry, composition, and physical process behind the measurement of CT numbers. The procedure starts with X-ray tube simulation and generation of PHSP file. The X-rays are then transported through CT and phantom inserts. Finally, a CT image is reconstructed and CT numbers are calculated [12].

The X-ray spectrum of the DURA 352-MV tube of the Siemens Emotion CT was input to the CT1 part of MC simulation. X-ray spectra were simulated using BEAMnrc/EGSnrc and the design information provided by Siemens. Optimization of the parameters of the $\mathrm{X}$-ray tube simulation was carried out in [21]. Simulations were performed for X-ray tube voltages of 80,100 , $105,110,115,120$ and $130 \mathrm{kV} .10^{8}$ incident electrons were used. The target was $0.2 \mathrm{~mm}$ tungsten. Total filtration was $0.6 \mathrm{~mm}$ titanium and $3.0 \mathrm{~mm}$ aluminium.

The X-ray tube was simulated as a collimated point source with a rectangular beam section. It was set $3.4 \mathrm{~cm}$ from the compound module simulating the filter. The filters, collimators and phantom with inserts were simulated as the compound modules: pyramids, blocks and side tubes, respectively [22]. However, as the BEAMnrc code terminates the history of particles outside the defined dimensions of the compound module, it was also necessary to simulate a side tube of 3 layers representing the tissue substitute, phantom and air surrounding the phantom in the field of view $(50 \mathrm{~cm}$ in diameter).

Output from the first part of the simulation (CT1-PHSP) served as input for the CT2 part of the simulation. Output for CT2 part of simulations was calculated at a scoring plane $94 \mathrm{~cm}$ from focal spot, which represented the position of the detector arrays. The detector array and the scatter grid were not simulated. Instead, ideal collimation was assumed and scattered photons were subtracted analytically (see below).

The incident number of photons in the CT1 part is $10^{10} .5 \times 10^{8}$ incident photons were used for the CT2 part of the simulation. If the number of photons in the CT1-PHSP was insufficient to generate the required $5 \times 10^{8}$ incident photons, the program was allowed to recycle the input file until the specified number of photons is achieved. The number of times that CT1-PHSP is recycled decreases when the peak voltage of the incident photon spectrum increases. The CT1-PHSP file was recycled 5 times with a voltage setting of $110 \mathrm{kV}$ to generate $5 \times 10^{8}$ incident photons for the CT2 simulation.

Each simulation of the CT2 part was regarded as an independent "view" i.e. the measurement at a single specified angle, which corresponds to a line in the sinogram. To generate more views using the same CT1-PHSP, the seeds of the random number generators were varied for CT2 part of the simulation.

Two Pentium4 Linux PC with $3 \mathrm{GHz}$ CPU power and 1 GB RAM were used to perform the MC simulation. The simulation of the CT1-PHSP with $10^{10}$ incident photons took several days of CPU time for each set of parameters. CT2 part of the simulation required a maximum of $3 \mathrm{~h}$ of CPU time for $5 \times 10^{8}$ incident photons.

\section{Postprocessing of simulation results}

\section{Sorting photons into detector elements}

Photons were initially sorted in the required slice thickness using the information on the position of the photons in the X-direction. Sorting the particles into detector elements along the slice-axis $(y$-axis of the simulation) required the knowledge of the number of detectors in the array $(N)$ and the length of the active part of the detector [22].

\section{Implementation of detector response}

The energy of each photon is given in the PHSP file. According to the energy of the photon, an efficiency factor (weight) is assigned to the photon. The weight is estimated from the energy response function of the detector elements [3]. The weights were calculated separately for each detector element in the array, and the result was then stored as list of the detector indices $(n)$ and the corresponding signal $(I(n))$.

\section{Scatter corrections}

Ideal collimation by the scatter grid was assumed. Scattered photons were excluded analytically based on the dimensions of the scatter-grid, the length of the active and dark areas of the detector and the incidence angle of each photon reaching the scoring plane. In a real CT scanner, particles that hit the active area of the detec- 
tors without hitting the scattering grid are considered "true events". The same concept was assumed in the simulation. Photons which were classified as scattered were not included in the signal $(I(n))$.

\section{Presenting projections in log format}

Calculation of projection data $\left(-\ln \left(I / I_{0}\right)\right)$, requires knowledge of the initial intensity of the incident photons $\left(I_{0}\right)$. Therefore, a dedicated simulation was set to calculate $I_{0}$ with only air in the FOV. $I_{0}(n)$ was calculated in the same manner as $I(n)$, and projections $(-\ln (I(n) /$ $\left.\left.I_{0}(n)\right)\right)$ were calculated for each detector element.

\section{Beam hardening corrections (BHC)}

Beam hardening is the shift of the X-ray spectrum to higher effective energies, which "harden" as the beam transverses matter. If the energy spectrum of the incident photons is known, beam hardening artefacts can be corrected. One approach is to assume the separation of variables of the attenuation coefficient $\mu(s, E)$, where $E$ is the energy and $s$ is the space. The details on this approach of BHC are described in [23, 24].

In this work, the energy assumed for BHC is $40 \mathrm{kV}$. Then, $\mu(s, E)$ is written as:

$$
\mu(s, E)=f(s) \cdot \psi(E)
$$

where $\psi(E)$ was the attenuation coefficient of water relative to the coefficient at $40 \mathrm{kV}$.

$$
\psi(E)=\mu_{\text {water }}(E) / \mu_{\text {water }}^{40 \mathrm{kV}}
$$

$\mathrm{BHC}$ is applied at the level of the raw data before reconstruction is performed. The $\mathrm{BHC}$ algorithm used in the scope of this work corrects the effect of beam hardening on the basis that all media interact with the spectrum similar to water ${ }^{2}$. This is a simple but sufficient approximation, since most tissue types are water-like. An approach that corrects for bone as well as soft tissues is described in [19].

\section{Reconstruction}

A simple fan-beam filtered back projection (FBP) reconstruction algorithm is applied to obtain images $[25]^{2}$. The reconstruction algorithm performs:

- Rebinning into parallel projections.

- Convolving with a Ram-Lak kernel.

- Back projecting.

The main input is the simulated projection. Other input parameters are the diameter of FOV, number of views, position of $\mathrm{CT}$ at starting angle, fan angle of the $\mathrm{X}$-ray tube and detectors, size of image and number of pixels per image. The output is an image matrix, which is given in arbitrary values. Though the values represent the distribution of the attenuation coefficients, they are not normalized.

${ }^{2}$ The code was provided by the image reconstruction group of the Medical Physics Department of the Institute of Medical Physics in the Freidrich-Alexander-University Erlangen-Nürnberg, Germany. The code was used with permission of developers for our specific calculations.

\section{Normalization of reconstructed values (Hounsfield units)}

Hounsfield units are the attenuation coefficients relative to $\mu_{\text {water }}$. To obtain $\mu_{\text {water }}$ a dedicated simulation is performed using a water cylinder ( $25 \mathrm{~cm}$ in diameter). The reconstructed value of $\mu_{\text {water }}\left(\mu_{\text {water }}^{\text {reconstucted }}\right)$ was calculated by defining a region of interest in the centre of the reconstructed image of water cylinder and calculating the average $\mu_{\text {water }}\left(\mu_{\text {water }}^{\text {recontructed }}\right)$.

The assumptions for normalization are that the reconstructed CT number of water $=0$, the reconstructed CT number of air is 1000 and that the relation between CT numbers and attenuation coefficients is linear. CT numbers were calculated using the reconstructed attenuation coefficient $\mu_{\text {water }}\left(\mu_{\text {water }}^{\text {recontructed }}\right)$ as:

$$
\text { CTnumber }=1000 \times\left(\frac{\mu_{m}^{\text {recon }}}{\mu_{w}^{\text {recon }}}-1\right) \mathrm{HU}
$$

\section{Results}

\section{CT numbers of the Gammex tissue substitutes, $\mathrm{H}$-materials and PMMA}

The measurements of CT numbers of Gammex tissue substitutes were performed using 80, 110 and $130 \mathrm{kV}$ nominal voltages. However, the actual voltage settings of the X-ray tube were $79.3,101.9$ and $121.7 \mathrm{kV}$.

The measurements performed by Jäkel et al. [15] indicated that H-materials behave differently from Gammex tissue substitutes, which closely imitate soft tissues. Figure 2 shows the calculated CT numbers as a function of the electron-density for Gammex tissue substitutes, $\mathrm{H}$-materials and PMMA. The results of simulation successfully predicted the behaviour of H-substitutes (compared to Gammex tissue substitutes).

The simulated CT numbers were calculated using 5 independent projections for the 80 and $120 \mathrm{kV}$ settings, but only one projection for the $100 \mathrm{kV}$. The effect of using one independent projection instead of five is a higher uncertainty in the simulated CT numbers of the simulations for the $100 \mathrm{kV}$ voltage settings.

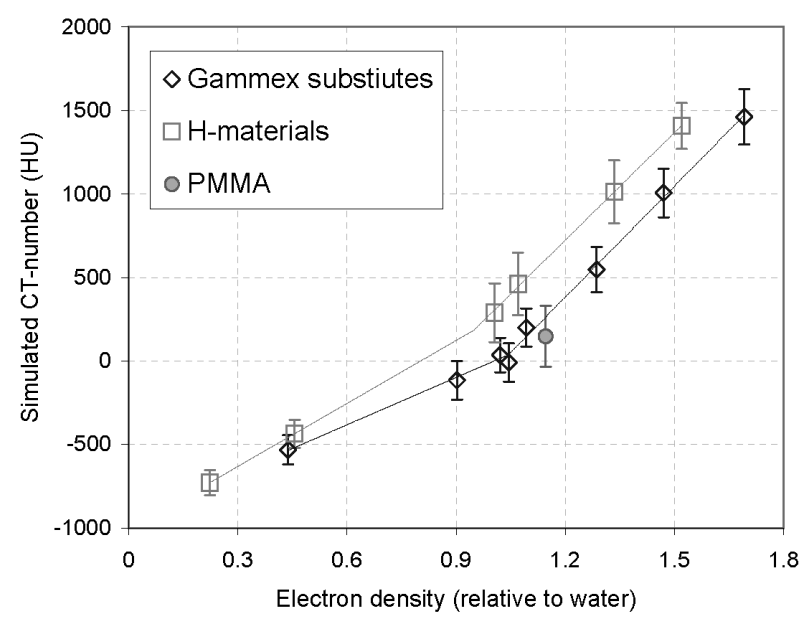

Fig. 2. Calculated CT numbers vs. electron density for Gammex, H-materials and PMMA. 

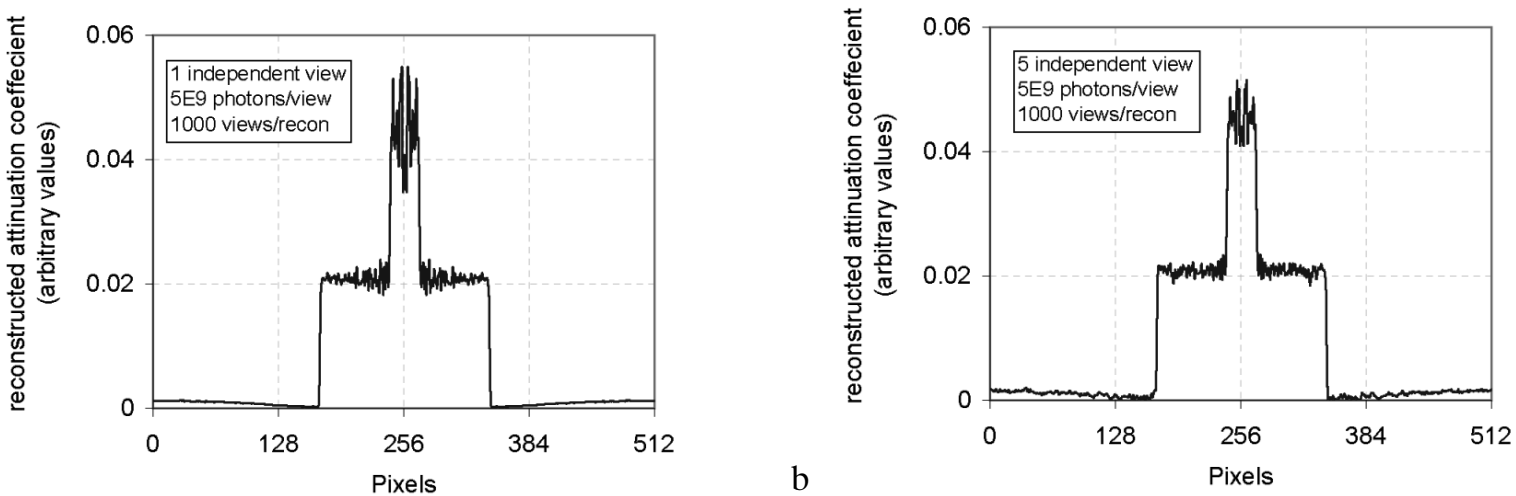

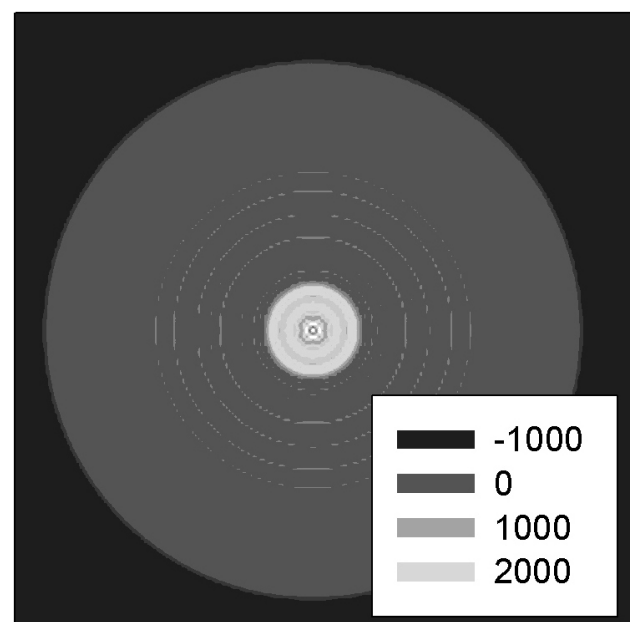

$\mathrm{b}$

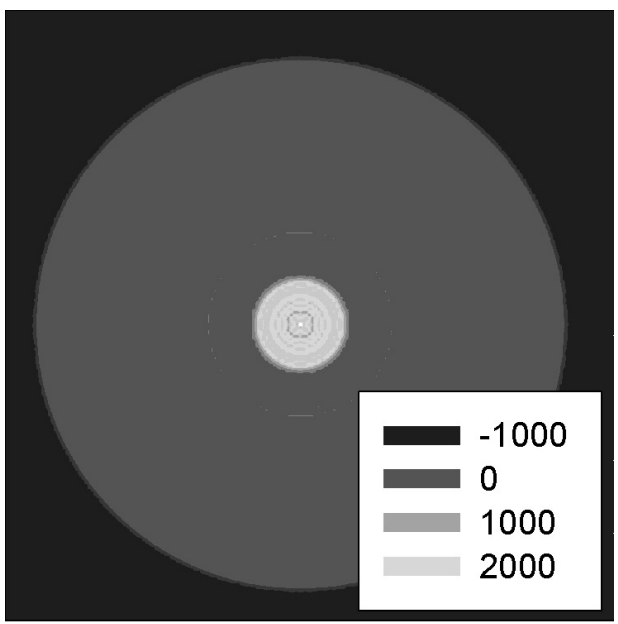

Fig. 3. The effect of number of independent projections on a profile in the centre of a calculated CT image (a and b) and the reconstructed images $(\mathrm{c}$ and $\mathrm{d})$ is presented. The reconstructed CT image $(512 \times 512$ pixels $)$ represents a $16 \mathrm{~cm}$ PMMA phantom with cortical bone substitute as an insert.

\section{Accuracy of the CT numbers}

The overall accuracy of the results was estimated taking into account the analysis of the statistical uncertainties of the calculated CT numbers and by comparison with experimental data.

\section{Uncertainty of the calculated CT numbers}

The photons in CT2-PHSP are dependent on the distribution of photons in CT1-PHSP. However, the use of different random number seeds for the simulations of the CT2 part cannot completely eliminate this dependency. The statistical effect of the number of independent views on the reconstructed image is investigated for a PMMA phantom with a Gammex cortical bone substitute as insert. Figure 3 is actually a horizontal profile through the centre of the image.

In Fig. 3a $\mu_{m}^{\text {recon }}$ was calculated with a single independent view compared to the 5 views shown in Fig. 3b. Despite the fact that the number of incident photons per view is the same, more fluctuations are seen in profile in Fig. 3a. The CT reconstructed images are shown in Figs. $3 c$ and 3d. Ring artefacts are visible in the image, which is reconstructed using one independent projection (Fig. 3c). The rings disappear when the image is reconstructed from 5 independent projections (Fig. 3d).

\section{Differences between measured and simulated CT numbers}

The deviation of the simulated CT numbers from the measured value was always within the uncertainty of the simulation. Figure 4 shows the simulated CT numbers as a function of measured CT numbers for Gammex tissue substitutes, H-materials and PMMA.

A histogram of all the data points from Table 2 is shown in Fig. 5. The simulation results are randomly dis-

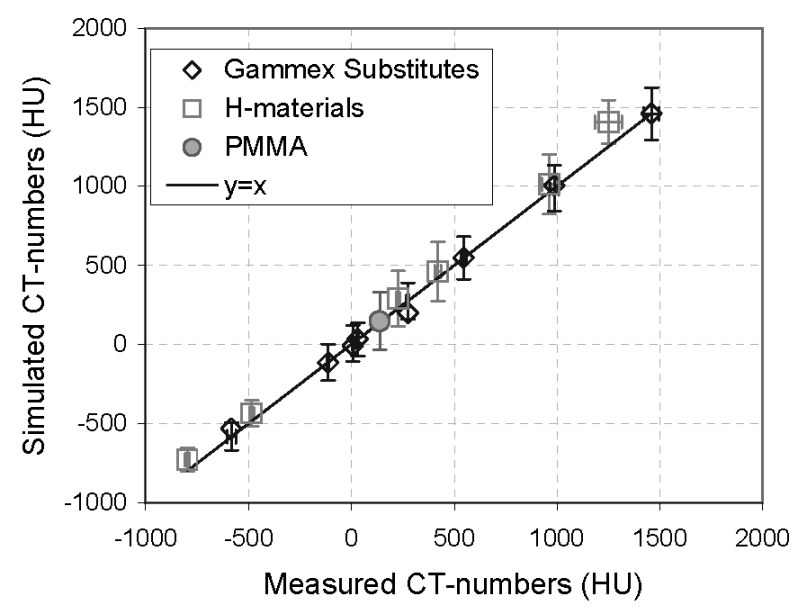

Fig. 4. Comparison of calculated and measured results of CT numbers for Gammex, H-materials and PMMA for the $120 \mathrm{kV}$ voltage setting. 
Table 2. Simulated and measured CT numbers for Gammex tissue substitutes, H-materials and PMMA

\begin{tabular}{|c|c|c|c|c|c|c|c|}
\hline & \multirow[b]{2}{*}{ E-density } & \multicolumn{3}{|c|}{ Measured CT numbers } & \multicolumn{3}{|c|}{ Simulated CT numbers } \\
\hline & & $\begin{array}{l}80 \mathrm{kV} \\
(79.3)\end{array}$ & $\begin{array}{l}110 \mathrm{kV} \\
(101.9)\end{array}$ & $\begin{array}{l}130 \mathrm{kV} \\
(121.7)\end{array}$ & $80 \mathrm{kV}$ & $100 \mathrm{kV}$ & $120 \mathrm{kV}$ \\
\hline$\overline{\text { Lung (LN450) }}$ & 0.44 & $-578 \pm 20$ & $-577 \pm 23$ & $-583 \pm 20$ & $-549 \pm 102$ & $-541 \pm 193$ & $-532 \pm 87$ \\
\hline Adipose fat (AP6) & 0.90 & $-137 \pm 4$ & $-121 \pm 4$ & $-114 \pm 4$ & $-143 \pm 106$ & $-140 \pm 220$ & $-116 \pm 115$ \\
\hline Brain (SR2) & 1.05 & $-17 \pm 4$ & $1 \pm 5$ & $7 \pm 4$ & $-2 \pm 150$ & $-46 \pm 235$ & $-10 \pm 115$ \\
\hline Muscle (RMI452) & 1.02 & $40 \pm 5$ & $35 \pm 5$ & $31 \pm 5$ & $44 \pm 91$ & $10 \pm 188$ & $35 \pm 103$ \\
\hline Inner bone(IB) & 1.09 & $391 \pm 9$ & $311 \pm 7$ & $274 \pm 6$ & $277 \pm 113$ & $220 \pm 189$ & $200 \pm 115$ \\
\hline CB2 30\% (CB230) & 1.29 & $723 \pm 16$ & $600 \pm 13$ & $546 \pm 11$ & $681 \pm 134$ & $556 \pm 256$ & $548 \pm 135$ \\
\hline CB2 $50 \%$ (CB250) & 1.47 & $1332 \pm 28$ & $1088 \pm 24$ & $987 \pm 22$ & $1253 \pm 97$ & $1042 \pm 428$ & $1006 \pm 145$ \\
\hline Cortical bone (SB3) & 1.69 & $1999 \pm 41$ & $1616 \pm 36$ & $1459 \pm 36$ & $1862 \pm 196$ & $1575 \pm 360$ & $1462 \pm 166$ \\
\hline $\mathrm{Hm} 800$ & 0.22 & - & - & $-798 \pm 11$ & - & - & $-729 \pm 75$ \\
\hline $\mathrm{Hm} 500$ & 0.46 & - & - & $-485 \pm 10$ & - & - & $-436 \pm 83$ \\
\hline H200 & 1.01 & - & - & $227 \pm 10$ & - & - & $289 \pm 176$ \\
\hline $\mathrm{H} 400$ & 1.07 & - & - & $420 \pm 15$ & - & - & $461 \pm 187$ \\
\hline H900 & 1.33 & - & - & $962 \pm 35$ & - & - & $1013 \pm 189$ \\
\hline H1200 & 1.52 & - & - & $1250 \pm 65$ & - & - & $1407 \pm 137$ \\
\hline$\underline{\text { PMMA }}$ & 1.15 & - & - & $138 \pm 7$ & - & - & $147 \pm 182$ \\
\hline
\end{tabular}

tributed around the measured values. The distribution can be described by the Gaussian function $(\mathrm{G})$ such that:

$$
\mathrm{G}(x)=A \cdot \exp \left(-\frac{(x-\bar{x})^{2}}{2 \sigma^{2}}\right)
$$

Here, $x$ is the difference between measured and calculated CT numbers; the average deviation $(\bar{x})$ is $4 \pm 4 \mathrm{HU}$. The standard deviation $(\sigma)$ is $49 \pm 4 \mathrm{HU}$ and $A$ is $12 \pm 1$.

\section{Discussion}

\section{Approximations in the simulation}

The simulation imitates the CT scanner, phantom and substitutes to the best available knowledge. However, the simulation setup included a few approximations, such as assuming that the X-ray tube is a point source, which were made in order to shorten the run-time of the simulation. Other approximations included the energy

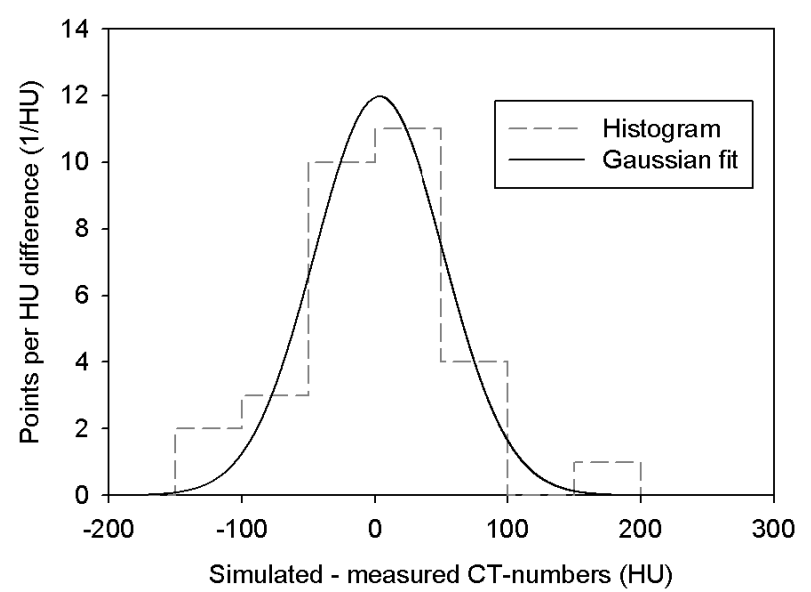

Fig. 5. Distribution of the simulated CT numbers around measured values. The graph shows the histograms of the data points as a function of the difference between the simulated and measured CT numbers. The histogram of all the data points and the Gaussian fit $(\mathrm{G}(x)$ in Eq. (5)) are shown. response of the detector, scatter and beam hardening corrections.

The beam profile due to the point-like X-ray source closely resembles the profiles obtained by the MC simulation of the X-ray tube. This approximation is valid only for the central slice (along the slice-plane) but only the central slices are of importance in this work. In general, the energy response of the detectors is mainly affected by photons with energy less than $52 \mathrm{kV}$ (the K-edge of Gd).

Influence of the applied correction of the signal due to scatter is analyzed in [22]. The shape of the scatter signal is similar to what was measured and predicted by Ay and Zaidi [26]. In our work, scatter was calculated by assuming perfect collimation by the anti-scatter grid. Scatter correction is time consuming and many photon counts are lost. However, without the scatter correction, the signal is more noisy and the value of CT numbers is underestimated.

In this work only water-based BHC was applied, as this was sufficient for simulation purposes. The deviation of the simulated CT numbers from the expected value (for example, $0 \mathrm{HU}$ for water) decreases by applying BHC. The difference between corrected and uncorrected CT numbers of water was less than $15 \mathrm{HU}$.

It should also be noted that $\mathrm{BHC}$ is a postprocessing correction that is applied to the simulated projections and does not increase the calculation time. It is recommended that, at minimum, a water-based $\mathrm{BHC}$ or even a weighted $\mathrm{BHC}$ be used in future simulations.

\section{Accuracy of the simulation}

The uncertainty of the simulated CT numbers depends on the simulated geometry and number of independent projections used to reconstruct the image (Fig. 3) as well as on the number of incident photons. Geometries causing large attenuations, such as large phantom diameters, result in the greatest uncertainties. If the attenuation is too large, the photons are absorbed within the phantom and barely reach the scoring plane. In such cases, it is 
necessary to simulate more photons in the CT2-part. However, increasing the number of photons in the CT2-part will lead to more recycling of the CT1-PHSP, which increases the uncertainty. The limiting factor is to obtain a sufficient number of photons (about $10^{6}$ per detector element) in order to reconstruct an image with reasonable quality.

In this work, images are reconstructed from a few independent projections. Therefore, it is important to have a high number of photon counts to generate projections with low statistical fluctuations.

Figure 3 shows the effect of statistical noise on the reconstructed attenuation coefficients in a profile through a reconstructed image. Usually, about 1000 independent views of approximately $10^{15}$ photons per view are used for imaging under clinical conditions. To simulate CT numbers in this work, a maximum of 5 independent views with only $5 \times 10^{8}$ photons per view were used. When the projections have a low signal to noise ratio, the reconstructed image have ring artefacts due to the correlation of the noise in the projections (Fig. 3).

To lower the uncertainty in the simulated CT numbers, it is necessary to simulate more photons and more independent projections. Therefore, it is necessary to increase the efficiency of the simulation.

A large number of incident photons used for the CT1-part of the simulation is lost by defining a $50 \times$ $50 \mathrm{~cm}^{2}$ field when only a $50 \times 1 \mathrm{~cm}^{2}$ field of X-ray is needed. This leads to long computation times of the CT1-simulations, since the run-time of the simulation increases with the number of incident photons. However, once a CT1-PHSP is available, it can be used repeatedly to perform CT2 simulations. BEAM is a flexible open-source code. It can model complex components; however, since it was designed to facilitate a simulation of a radiotherapy unit, it has a few drawbacks when used to simulate a CT scanner. The code was nonetheless sufficient for the purpose we wanted to achieve. However, the EGSnrc or MCNP codes may be more useful for introducing asymmetric geometries in CT scanner and phantom simulations.

Our simulation correctly reproduced the behaviour of H-materials compared to Gammex tissue substitutes and the effect of voltage settings of the X-ray tube. The simulations of the $\mathrm{CT}$ numbers of $\mathrm{H}$-materials were based on the Emotion CT scanner despite the fact that it is different from the Siemens Somatom Plus 4 scanner used by Jäkel et al. [15]. However, both scanners are from Siemens and are of comparable design-generations. This explains the remarkable result that the average deviation between measured and simulated CT numbers was $4 \pm 4 \mathrm{HU}$ with standard deviation $\sigma=49 \pm 4$ HU. The difference between the measured and simulated CT numbers is small and always within the uncertainty of the simulated CT numbers.

\section{Conclusion}

This work has described a novel method for calculating CT numbers from MC simulations, and is the first study of the accuracy of CT numbers based on MC simulations of a CT scanner. The average deviation between measured and simulated CT numbers is $4 \pm 4 \mathrm{HU}$, with a standard deviation $\sigma=49 \pm 4 \mathrm{HU}$. This is a remarkable result, particularly since the uncertainty of simulated CT numbers can rise to $200 \mathrm{HU}$ or more, depending on the simulation parameters.

This method was also used to investigate the suitability of tissue substitutes to perform CT calibrations. For example, $\mathrm{H}$-materials were found unsuitable to perform CT calibrations, whereas Gammex tissue substitutes closely resemble the behaviour of body tissues. This simulation correctly predicted the CT numbers of $\mathrm{H}$-material and distinguished them from Gammex tissue substitutes.

The method presented here can correctly assess CT numbers for cylindrical objects in the centre of the FOV. However, oval, rectangular or asymmetric objects, which cannot be approximated by a few independent views, have not yet been investigated.

The method presented here could be used to estimate the effect of different parameters on CT numbers, for example, the voltage of the X-ray tube, the size and material of the phantom. Future work will discuss how these parameters affect range calculation in ion therapy treatment planning.

The uncertainties in the empirical calibration of the CT-numbers described by us in [27] inspired us to develop the methodology presented here, but there are different ways to improve the accuracy of CT-numbers [28] and empirical calibration [29] and these are briefly discussed in [30].

Acknowledgment. Prof. Dr. M. Kachelriess and Dr. C. Penssel, from the Institute of Medical Physics in Erlangen (Germany) provided the BHC and the reconstruction code. Dr. S. Ulzheimer, Dr. H. Wallschläger and Dr. B. J. Heismann, from Siemens Medical Solutions, provided technical data on the Emotion CT, X-ray spectra and the response function of the detectors. M. Romanchikova provided most of the simulated X-ray spectra.

\section{References}

1. Mustafa, A. A., \& Jackson, D. F. (1983). The relation between X-ray CT numbers and charged particle stopping powers and its significance for radiotherapy treatment planning. Phys. Med. Biol., 28, 169-176.

2. Verhaegen, F., \& Devic, S. (2005). Sensitivity study for CT images in Monte Carlo treatment planning. Phys. Med. Biol., 50, 937-946.

3. Homolka, P., Gahleitner, A., \& Nowotny, R. (2002). Temperature dependence of HU values for various water equivalent phantom materials. Phys. Med. Biol., 47, 2917-2923.

4. Bhat, M., Pattison, J., Bibbo, G., \& Caon, M. (1998). Diagnostic X-ray spectra: a comparison of spectra generated by different computational methods with a measured spectrum. Med. Phys., 25, 114-120.

5. Caon, M., Bibbo, G., Pattison, J., \& Bhat, M. (1998). The effect on dose to computed tomography phantoms of varying the theoretical X-ray spectrum: a comparison of four diagnostic spectrum calculating codes. Med. Phys., 25, 1021-1027.

6. Ay, M. R., Sarkar, S., Shahriari, M., \& Zaidi, H. (2005). Assessment of different computational models for generation of X-ray spectra in diagnostic radiology and mammography. Med. Phys., 32, 1660-1675. 
7. Ay, M. R., Shahriari, M., Sarkar, S., \& Zaidi, H. (2004). Monte Carlo simulation of X-ray spectra in diagnostic radiology and mammography using MCNP4C. Phys. Med. Biol., 49, 4897-4917.

8. Atherton, J. V., \& Huda, W. (1995). CT dose in cylindrical phantoms. Phys. Med. Biol., 40, 891-911.

9. Jarry, G., DeMacro, J. J., Beifuss, U., \& Cagnon, C. H. (2003). A Monte Carlo-based method to estimate radiation dose from spiral CT: from phantom testing to patient-specific models. Phys. Med. Biol., 48, 2645-2663.

10. Salvado, M., Lopez, M., Morant, J. J., \& Calzado, A. (2005). Monte Carlo calculations of radiation dose in CT examination using phantom and patient tomographic models. Radiat. Prot. Dosim., 114, 364-368.

11. Tzedakis, A., \& Perisnakis, K. (2006). The effect of Z overscanning on radiation burden of pediatric patients undergoing head CT with multidetector scanners: A Monte Carlo study. Med. Phys., 33(7), 2472-2478.

12. Wysocka-Rabin, A., Qamhiyeh, S., \& Jäkel, O. (2011). Simulation of computed tomography (CT) images using a Monte Carlo approach. Nukleonika, 56(4), 299-304.

13. Heismann, B. J., Leppert, J., \& Stierstorfer, K. (2003). Density and atomic number measurements with spectral X-ray attenuation method. J. Appl. Phys., 94, 2073-2079.

14. Gammex-RMI. (2004). Electron density CT phantom. Catalogue. Retrieved from http://www.gammex.com/ ace-files/Gammex_Catalog.pdf.

15. Jäkel, O., Jacob, C., Schardt, D., Karger, C., \& Hartmann, G. H. (2001). Relation between carbon ion ranges and X-ray CT numbers. Med. Phys., 28(4), 701-703.

16. Kawrakow, I. (2000). Accurate condensed history Monte Carlo simulation of electron transport. EGSnrc, the new EGS4 version. Med. Phys., 27, 485-498.

17. Kawrakow, I., \& Rogers, D. W. O. (2003). The EGSnrc cod system: Monte Carlo simulation of electron and photon transports. Ottawa: National Research Council of Canada. (PRIS-701).

18. Rogers, D. W. O., Ma, C. M., Walters, B., Ding, G. X., Sheikh-Bagheri, D., \& Zhang, G. (2001). BEAMnrc Users manual. Ottawa: National Research Council of Canada. (PRIS-0509(A) rev. G).

19. Verhaegen, F. (2002). Evaluation of the EGSnrc Monte Carlo code for interference near high- $\mathrm{Z}$ media exposed to kilovolt and ${ }^{60}$ Co photons. Phys. Med. Biol., 47, 1691-1705.
20. Verhaegen, F., Nahum, A. E., Van de Putte, S., \& Namito, Y. (1999). Monte Carlo modelling of radiotherapy kV X-ray units. Phys. Med. Biol., 44, 1767-1789.

21. Romanchikova, M. (2006). Monte Carlo Simulation des Röntgenspektrums einer computertomographischen Röntgenröhre. Unpublished Master's thesis, University of Heidelberg, Germany.

22. Qamhiyeh, S. (2007). A Monte Carlo study of the accuracy of CT numbers for range calculations in Carbon ion therapy. Unpublished $\mathrm{PhD}$ thesis, University of Heidelberg, Germany.

23. Kachelrieß, M., \& Kalender, W. (2005). Improving PET/ $\mathrm{CT}$ attenuation correction with iterative CT beam hardening corrections. In 2005 IEEE Nuclear Science Symposium Conference Record, 23-29 October 2005. (Vol. 4). IEEE. DOI: 10.1109/NSSMIC.2005.1596704.

24. Kachelrieß, M., Sourbelle, K., \& Kalender, W. (2006). Empirical cupping corrections: a first-order raw data precorrection for cone beam computed tomography. Phys. Med. Biol., 33, 1269-1274.

25. Sennst, D. A., Kachelriess, M., Leidercker, C., Schmidt, B., Watzke, O., \& Kalender, W. A. (2004). An extensible software-based platform for reconstruction and evaluation of CT images. Radiographics, 24(2), 601-613.

26. Ay, M. R., \& Zaidi, H. (2005). Development and validation of MCNP4C-based Monte Carlo simulator for fan and cone beam X-ray CT. Phys. Med. Biol., 50, 4863-3885.

27. Qamhiyeh, S., Wysocka-Rabin, A., Ellerbrock, M., \& Jäkel, O. (2007). Effect of voltage of CT scanner, phantom size and phantom material on CT calibration and carbon range. Radiother. Oncol., 84(S1), S232.

28. Bazalova, M., Carrier, J. F., Beaulieu, L., \& Verhaegen, F. (2008). Tissue segmentation in Monte Carlo treatment planning: a simulation study using dual-energy CT images. Radiother. Oncol., 86(1), 93-98.

29. Hünemohr, N., Krauss, B., Dinkel, J., Gillmann, C., Ackermann, B., Jäkel, O., \& Greilich, S. (2013). Ion range estimation by using dual energy computed tomography. Z. Med. Phys., 23(4), 300-313.

30. Wysocka-Rabin, A. (2013) Advances in conformal radiotherapy using Monte Carlo Code to design new IMRT and IORT Accelerators and interpret CT numbers. (CERN-WUT Editorial series on "Accelerator Science". Vol. 17). Warsaw: Institute of Electronic Systems, Warsaw University of Technology. 